\title{
Use of an In Vitro Skin Parallel Artificial Membrane Assay (Skin-PAMPA) as a Screening Tool to Compare Transdermal Permeability of Model Compound 4-Phenylethyl-Resorcinol Dissolved in Different Solvents
}

\author{
Bálint Sinkó ${ }^{1,2}$, Vivien Bárdos ${ }^{1}$, Dániel Vesztergombi ${ }^{1}$, Szabina Kádár ${ }^{3}$, Petra Malcsiner ${ }^{1}$, Anne Moustie ${ }^{4}$, \\ Chantal Jouy ${ }^{4}$, Krisztina Takács-Novák ${ }^{1, *}$ and Sebastien Grégoire ${ }^{4}$
}

check for updates

Citation: Sinkó, B.; Bárdos, V.; Vesztergombi, D.; Kádár, S.; Malcsiner, P.; Moustie, A.; Jouy, C.; Takács-Novák, K.; Grégoire, S. Use of an In Vitro Skin Parallel Artificial Membrane Assay (Skin-PAMPA) as a Screening Tool to Compare Transdermal Permeability of Model Compound 4-Phenylethyl-Resorcinol Dissolved in Different Solvents. Pharmaceutics 2021, 13, 1758. https:// doi.org/10.3390/pharmaceutics 13111758

Academic Editors: Ana Melero, Ruy Carlos Ruver Beck and Heather Benson

Received: 7 September 2021 Accepted: 18 October 2021 Published: 21 October 2021

Publisher's Note: MDPI stays neutral with regard to jurisdictional claims in published maps and institutional affiliations.

Copyright: (c) 2021 by the authors. Licensee MDPI, Basel, Switzerland. This article is an open access article distributed under the terms and conditions of the Creative Commons Attribution (CC BY) license (https:/ / creativecommons.org/licenses/by/ $4.0 /)$.
1 Department of Pharmaceutical Chemistry, Semmelweis University, H-1092 Budapest, Hungary; bsinko@pion-inc.com (B.S.); bardos.vivien@phd.semmelweis.hu (V.B.); vesztdani@gmail.com (D.V.); malcsiner.petra@gmail.com (P.M.)

2 Pion Inc., Billerica, MA 01821, USA

3 Department of Organic Chemistry and Technology, Budapest University of Technology and Economics, H-1111 Budapest, Hungary; kadar.szabina@vbk.bme.hu

4 L'Oréal Research \& Innovation, 93601 Aulnay-sous Bois, France; anne.moustie@rd.loreal.com (A.M.); chantal.jouy@rd.loreal.com (C.J.); sebastien.gregoire@rd.loreal.com (S.G.)

* Correspondence: novak.krisztina@pharma.semmelweis-univ.hu; Tel.: +33-3612155241

\begin{abstract}
Absorption through the skin of topically applied chemicals is relevant for both formulation development and safety assessment, especially in the early stages of development. However, the supply of human skin is limited, and the traditional in vitro methods are of low throughput. As an alternative, an artificial membrane-based Skin Parallel Artificial Membrane Permeability Assay (Skin-PAMPA) has been developed to mimic the permeability through the stratum corneum. In this study, this assay was used to measure the permeability of a model compound, 4-phenylethylresorcinol (PER), dissolved in 13 different solvents that are commonly used in cosmetic formulation development. The study was performed at concentrations close to the saturated solution of PER in each solvent to investigate the maximum thermodynamic potential of the solvents. The permeability of PER in selected solvents was also measured on ex vivo pig skin for comparison. Pig ear skin is an accepted alternative model of human skin. The permeability coefficient, which is independent of the concentration of the applied solution, showed a good correlation $\left(R^{2}=0.844\right)$ between the Skin-PAMPA and the pig skin permeation data. Our results support the use of the Skin-PAMPA to screen the suitability of different solvents for non-polar compounds at an early stage of formulation development.
\end{abstract}

Keywords: Skin-PAMPA; formulation; skin barrier; permeability; pig skin; safety testing

\section{Introduction}

Absorption through the skin of topically applied chemicals (e.g., drugs, cosmetics, iatrogenic substances) is relevant for both formulation development and safety assessment [1,2]. In the pharmacological domain, transdermal drug delivery offers multiple advantages over oral or parenteral administrations (e.g., by-passing "first-pass" metabolism, providing sustained drug release, protection of the GI tract from drugs, fewer side effects) [3]. In the cosmetic industry, the safety assessment of ingredients requires an estimation of their local and systemic exposure(s) when applied topically. Guidelines define clear criteria to conduct such skin absorption studies and point out in vitro human skin as the gold standard for study or pig skin as an alternative [4,5]. Although many data are available in these guidelines, the quality and reproducibility of the data are related to the assay criteria defined in the guidelines (skin preparation, receptor fluid chosen, skin 
test integrity, etc.) [6,7] and also to the validation of the analytical methods [8]. As the evaluation of skin penetration of compounds is needed at an early stage of development, such skin absorption study on ex vivo human skin is not suitable. As an alternative model, reconstructed skin has been utilized [9], with some limitations on the reproducibility and prediction capacity [10]. Alternatively, synthetic membrane models have been developed to mimic the main features of the stratum corneum (SC) [11-15], which acts as a rate-limiting barrier [16]. These membranes are easily available and are more cost-effective than ex vivo human skin. Moreover, it has been already demonstrated that such models can be successfully used in an initial screening approach to assist formulation selection before a more biological model is involved $[17,18]$.

Recently, an artificial membrane-based in vitro method, the Skin Parallel Artificial Membrane Permeability Assay (Skin-PAMPA), was developed in a 96-well plate format [15]. Such layout is suitable for automation as well as high-throughput screening. This SkinPAMPA model has been shown to possess a high prediction capability [19] not just for buffer based sample solutions, but also for both semisolid formulations (gel, ointment and cream) $[20,21]$ and transdermal patches [22].

In product formulation, various vehicles are designed to modulate skin absorption by altering the solubility and permeability of an active ingredient. Penetration across the SC involves interactions among the solvent(s), SC and the active ingredient. Even if an artificial membrane cannot mimic SC in its overall complexity, it could be used to investigate the effect of solvent itself. Therefore, this project aimed to investigate the applicability of the Skin-PAMPA model on a wide range of safe "solvents" traditionally used by the cosmetic industry. In the study, the permeation of a model compound, 4phenylethyl-resorcinol (PER) (see structure in Table 1), a skin-lightening agent used both in cosmetic and dermatologic formulations, was tested on the Skin-PAMPA model in 13 solvents (9 pure solvents and 4 simple mixtures, coded as S1-S13 in Table 2). The model compound was selected based on three aspects: (i) physico-chemical properties, (ii) solubility in a wide range of relevant solvents (to some degree) and (iii) good UV absorption to make the direct UV spectroscopy possible. PER is a non-polar, weak acid that is neutral at physiological $\mathrm{pH}$. It has suitable UV properties and reasonable solubility in the solvents examined, which made it a good model for the study. A recently published study from Zhang and co-workers [23] has reported a comprehensive characterisation of PER, including HPLC-based $\log P$ and solubility and in vitro permeation studies through human and porcine skin. The permeation profile of PER was investigated in finite dose conditions using Franz diffusion cell method and applying PER in three different vehicles. The study concluded that the properties of PER make it a suitable compound for dermal delivery, which also confirms our selection of PER as model compound for this study.

Table 1. Chemical structure and physico-chemical properties of PER.

\begin{tabular}{|c|c|}
\hline \multicolumn{2}{|c|}{ PER } \\
\hline \multicolumn{2}{|l|}{ Structure } \\
\hline Chemical Name & 4-phenylethyl-resorcinol \\
\hline CAS Number & $94-77-9$ \\
\hline Molecular Weight & $214.3(\mathrm{~g} / \mathrm{mol})$ \\
\hline $\log P$ & $2.98^{1}$ \\
\hline$p K_{a}$ & 9.77-10.77 (AH/ $\left.\mathrm{A}^{-}\right)^{2}$ \\
\hline Solubilty in Prisma Buffer & $3.45^{1}(\mathrm{mg} / \mathrm{mL})$ \\
\hline Water Solubility & $3.85(\mathrm{mg} / \mathrm{mL})$ \\
\hline
\end{tabular}

${ }^{1}$ data measured at Semmelweis University; ${ }^{2}$ data obtained from L'Oréal Laboratories. 
The aqueous solubility of PER was measured and compared with available data. To have comparable results between the different solvents used, PER was solubilized at saturation in tested solvents. Infinite conditions were used for all experiments, as this allowed measuring typical parameters describing percutaneous absorption [24]: permeability coefficient ( $P_{m}$ for PAMPA and $K_{p}$ for pig skin permeation), flux $(J)$ and the amount penetrated in a finite time $(Q t)$. To identify the best parameter to differentiate between the percutaneous absorption of PER in different solvents and to validate the Skin-PAMPA, the samples were also tested in a pig skin model. This method was suggested as a suitable alternative to human skin by the Scientific Committee on Consumer Safety [4]. Since pig skin penetration assays are resource- and time-consuming, it was not possible to measure the penetration of PER in all solvents. Hence, a limited number of solvents (9 out of 13) were tested spanning different types and solubility. In addition, not all formulations were suitable for this assay, since a sufficiently high concentration could not be achieved, due to the low solubility (S11, S12, S13).

Table 2. Solvents used in the study and the solubility of PER in different solvents.

\begin{tabular}{|c|c|c|c|c|c|}
\hline Solvent Class & Code & Solvent & MW & $\begin{array}{c}\text { PER } \\
\text { Approximate } \\
\text { Solubility } \\
(\mathrm{mg} / \mathrm{mL})\end{array}$ & $\begin{array}{c}\text { PER } \\
\text { Equilibrium } \\
\text { Solubility }^{2} \\
\text { (mg/mL) }\end{array}$ \\
\hline \multirow{6}{*}{$\begin{array}{l}\text { Low-MW polar } \\
\text { solvents }\end{array}$} & S1 & \multirow{6}{*}{$\begin{array}{c}\text { Water } \\
\text { Ethanol } \\
\text { Glycerol } \\
\text { Dimethylisosorbide } \\
\text { Water/ethanol 80:20 }(w / w) \\
\text { Water/dimethylisosorbide } \\
90: 10(w / w)\end{array}$} & 18.0 & 1 & $1.3 \pm 0.2$ \\
\hline & S2 & & 46.1 & $>1000$ & $368 \pm 52$ \\
\hline & S3 & & 92.1 & 5 & - \\
\hline & $\mathrm{S} 4$ & & 174.2 & 75 & $60 \pm 5.7$ \\
\hline & S5 & & NA & 10 & $8.1 \pm 4.3$ \\
\hline & S6 & & NA & 1 & $1.1 \pm 0.1$ \\
\hline \multirow{3}{*}{$\begin{array}{l}\text { Low-MW polar } \\
\text { "glycol" solvents }\end{array}$} & S7 & \multirow{3}{*}{$\begin{array}{c}\text { Propylene glycol } \\
\text { Water:propylene glycol } \\
\text { 80:20 }(w / w) \\
\text { Water propylenglycol/ethanol } \\
\text { 10:30:60 }(w / w / w)\end{array}$} & 76.1 & 500 & $350 \pm 21$ \\
\hline & S8 & & NA & 10 & $5.1 \pm 0.8$ \\
\hline & S9 & & NA & $>1000$ & $373 \pm 49$ \\
\hline \multirow{4}{*}{$\begin{array}{c}\text { High-MW } \\
\text { non-polar solvents }\end{array}$} & S10 & $\begin{array}{c}\text { Capric/caprylic } \\
\text { triglycerides }\end{array}$ & $554.8 / 470.7$ & 75 & $74 \pm 5.1$ \\
\hline & S11 & Octyl dodecanol & 298.6 & 1 & - \\
\hline & S12 & Apricot kernel oil & NA & 1 & - \\
\hline & S13 & Corn oil & NA & 1 & - \\
\hline
\end{tabular}

${ }^{1}$ Approximate solubility of PER determined by semi-quantitative method [25], ${ }^{2}$ equilibrium solubility ( $\left.S_{\mathrm{o}}\right)$ measured with LC/MS/MS for the pig skin studies.

\section{Materials and Methods}

\subsection{Materials}

All solvents were provided by the French L'Oréal Laboratories. PER (CAS 94-77-9) was obtained from Symrise ${ }^{\mathrm{TM}}$ (Table 1). Lucinol (CAS 18979-61-8) used as internal standard for LC/MS-MS PER quantification was provided by L'Oreal. The applied concentrations of PER in different solvents are shown in Table 2. For sake of simplicity, the solvents are referred to in the text using a code system S1-S13. All the other reagents were of analytical grade and purchased from Sigma-Aldrich (Lyon, France) or Reanal ${ }^{\mathrm{TM}}$ (Budapest, Hungary). Pig ear skin was obtained from a slaughterhouse (Pouldreuzic, France), frozen at $-20{ }^{\circ} \mathrm{C}$ after sampling and stored prior to use.

\subsection{Solubility Measurements}

As a first step, the solubility class at $32{ }^{\circ} \mathrm{C}$ (i.e., the temperature of the human skin surface) was determined according to the OECD test guideline No. 105 [25]. In a stepwise procedure, increasing volumes of the given solvent (pre-warmed at $32{ }^{\circ} \mathrm{C}$ ) were added to precisely weighted amount $0.1 \mathrm{~g}$ of the PER sample in a $10 \mathrm{~mL}$ glass-stoppered measuring cylinder. After each addition of the solvent aliquots, the mixture was shaken for $10 \mathrm{~min}$ and evaluated visually for any undissolved particles of the solid. When, after addition of $10 \mathrm{~mL}$ of solvent, the sample remained undissolved, the experiment was continued in 
a $100 \mathrm{~mL}$ cylinder. The approximate solubility is given as the volume of the solvent in which complete dissolution was observed after $1 \mathrm{~h}$. The sample was then stirred for $24 \mathrm{~h}$ before a final visual assessment. Based on this method, five solubility categories were set between 1 and $1000 \mathrm{mg} / \mathrm{mL}$. A further refinement step included four subclasses in each category. For example, if the compound was soluble in the $1-10 \mathrm{mg} / \mathrm{mL}$ category, the solution was checked at concentrations of $2.5,5,7.5$ and $10 \mathrm{mg} / \mathrm{mL}$ to determine the closest value to the saturated solution. These solubility categories defined the concentrations in the permeability test, which had a maximum of $500 \mathrm{mg} / \mathrm{mL}$ ( $50 \%$ of the maximum solubility category: $1000 \mathrm{mg} / \mathrm{mL}$ ). For solvents tested on pig skin, solutions used were analysed by LC/MS/MS. For this purpose, a solution at an upper limit of the solubility class previously defined was prepared and centrifuged at $14,000 \mathrm{rpm}$ to guarantee particle precipitation before analysis.

The equilibrium intrinsic solubility value of model compound in the acceptor medium was determined by the standardized protocol of saturation shake flask method $[26,27]$. The measurements were carried out at a controlled temperature $32.0 \pm 0.5^{\circ} \mathrm{C}$. The sample was added to $5 \mathrm{~mL}$ of Prisma buffer solution $\mathrm{pH} 7.4$ (which served as the acceptor phase in PAMPA experiments) until a heterogeneous system (solid sample and liquid) was obtained. The solubility suspension containing solid excess of the sample was stirred for a period of $6 \mathrm{~h}$ (stirring time) followed by $18 \mathrm{~h}$ of sedimentation to achieve the thermodynamic equilibrium. After sedimentation and the necessary dilution, the concentration of the saturated solution was measured by UV spectroscopy. The solubility experiments were performed in triplicate.

\section{3. $\log P$ Measurement}

The $\log P$ value of PER was measured in octanol/water system at $25.0 \pm 0.1^{\circ} \mathrm{C}$ by standard shake-flask method described in our former papers [28,29]. Two parallel experiments were carried out.

Four different phase ratios of octanol:water (1:50, 1:75, 1:100, 1:125) were applied. The equilibration time was $1 \mathrm{~h}$ (Lauda M2OS, shaking thermostat Königshofen, Germany), and the phases were separated by centrifugation. The concentration decrease in the sample in the aqueous phase was detected by UV spectroscopy (Jasco V-550 UV/VIS spectrophotometer, Easton, MD, USA) measuring the absorbance before and after the partition at $\lambda_{\max }=280 \mathrm{~nm}$. The $\log P$ value was calculated from the equation:

$$
\log P=\log \left[\frac{A_{0}-A_{1}}{A_{1}}\left(\frac{V_{a q}}{V_{o c t}}\right)\right]
$$

where $A_{0}$ and $A_{1}$ represent the absorbance value at the absorption maximum of the compound in the aqueous phase before and after partition [28].

\subsection{Permeability Measurements Using Skin-PAMPA Plates}

Membrane permeability of PER was measured using commercially available SkinPAMPA plates (Skin-PAMPA ${ }^{\mathrm{TM}}$, Pion Inc., Billerica, MA, USA). Skin PAMPA ${ }^{\mathrm{TM}}$ sandwiches and stirring bars (P/N: 110211) were supplied by Pion Inc ${ }^{\mathrm{TM}}$. UV plates (UV-star microplate, clear, flat bottom, half area) were from Greiner Bio-one ${ }^{\mathrm{TM}}$ (Kremsmünster, Austria). Membranes were hydrated overnight with standard hydration solution (Pion Inc ${ }^{\mathrm{TM}}$., product number 120706). The donor phase solutions of PER in different solvents were prepared freshly according to the approximate solubility (Table 2), and $70 \mu \mathrm{L}$ (corresponding to $233 \mu \mathrm{L} / \mathrm{cm}^{2}$ for $0.3 \mathrm{~cm}^{2}$ exposure area) was applied to the donor (upper) plate. The acceptor (lower) plate contained $180 \mu \mathrm{L}$ Prisma buffer $\mathrm{pH} 7.4$ and a magnetic stirrer in each well. The PAMPA ${ }^{\mathrm{TM}}$ sandwich was incubated at $32^{\circ} \mathrm{C}$ in a Gut-Box ${ }^{\mathrm{TM}}$ (from Pion Inc ${ }^{\mathrm{TM}}$ ). Stirring bars were applied in every well to avoid the effect of the unstirred water layer. The acceptor solution was sampled after 7.5, 15, 30, 60, 120, 240 and 360 min incubation. After each individual incubation period, $150 \mu \mathrm{L}$ from the acceptor compartment was transferred to UV plates. The acceptor phase was replaced with fresh buffer solution. UV absorption 
was measured at $\lambda=280 \mathrm{~nm}$ (Tecan Infinite M200 UV-plate reader driven by Magellan v.7.2. software (Tecan ${ }^{\mathrm{TM}}$, Männedorf, Switzerland) after dilution if necessary, and the concentration of PER was calculated using the calibration curve $\mathrm{A}=117.95 c+0.01\left(R^{2}=0.9997\right.$, $n=9$ ), in the concentration range $9-90 \mu \mathrm{g} / \mathrm{mL}$.

Parameters characterizing the transdermal penetration were obtained from the cumulative amount of PER penetrated per $\mathrm{cm}^{2}$ versus time plots. The flux $(J)$ was obtained as the slope of the permeability profile and expressed in $\mu \mathrm{g} / \mathrm{cm}^{2} \times \mathrm{h}$ units. For the linear regression analysis, the linear range of incubation period from 0 to $30 \mathrm{~min}$ was selected and used for calculation of flux of the model compound. Permeability coefficient $P_{m}$ $\left(\mathrm{cm}^{-2} \times \mathrm{h}^{-1}\right)$ was calculated from the equation:

$$
P_{m}=J / C_{D}
$$

where $C_{D}$ is the donor phase concentration.

The area under the curve $(A U C)$ was calculated by integration of the permeability profile between 0 and $6 \mathrm{~h}$ using OriginPro v.2019b (OriginLab Corporation, Northampton, MA, USA).

\subsection{Skin-PAMPA Membrane Integrity Study}

Possible disruption by solvents of the integrity of the biomimetic artificial membrane was investigated. Wells were filled with each solvent and incubated over a longer (minimum $7 \mathrm{~h}$ ) incubation time than the duration of the tests with the model solutions. The solvents were aspirated from the wells, and the residue from the surface of the membrane was removed gently with cotton paper. A standard skin permeability assay was then performed using piroxicam as the model permeant, for which precise previous data are available [22]. The $\log P_{m}$ values were compared with the reference value from untreated plates.

\subsection{Penetration Kinetics across Pig Ear Skin}

Before use, hairs were shaved from the pig ear skin using an electric razor, and the skin thickness was adjusted between 700 and $1200 \mu \mathrm{m}$. This size range was achieved by cutting the dermis below hair follicle. The integrity of the skin was tested according to the Trans-Epidermal Water Loss (TEWL) method using a Delfin device. The TEWL of dermatomed skin was always lower than $15 \mathrm{~g} / \mathrm{m}^{2} \times \mathrm{h}$ (cut-off value was defined according to historical date obtained in the lab), indicating that storage at $-20{ }^{\circ} \mathrm{C}$ and dermatome did not compromise skin integrity. The number of discs per treatment was between 2 and 10 replicates.

After topical application of the test chemical (infinite dose, $1.13 \mathrm{~mL} / \mathrm{cm}^{2}$ ), the concentration of the chemical in the receptor fluid was measured by sampling $200 \mu \mathrm{L}$ of receptor fluid and replacing it with fresh fluid on an hourly basis, up to $16 \mathrm{~h}$. The receptor fluid selected for PER was sodium chloride solution $(9 \mathrm{~g} / \mathrm{L})$ supplemented with $0.25 \%$ $(v / v)$ Tween 80 .

The kinetic samples were directly injected into an LC/MS-MS system (Shimadzu Nexera LC system, Shimadzu, Kyoto, Japan) coupled with a mass spectrometer API 3500 (Sciex, Framingham, MA, USA). The analytical system was managed by Analyst v.1.6 software (Sciex, Framingham, MA, USA). The analytical column used was a Kinetex C18 from Phenomenex ${ }^{\mathrm{TM}}$ (Torrance, CA, USA) $(50 \times 2.0 \mathrm{~mm}$, dp. $2.6 \mu \mathrm{m})$, and analysis was carried out with a gradient elution with mobile phases of $20 \mathrm{mM}$ ammonium acetate (A) and acetonitrile (B). The column temperature was fixed at $50{ }^{\circ} \mathrm{C}$, and the volume of the injection was $10 \mu \mathrm{L}$ with a flow rate of $0.8 \mathrm{~mL} / \mathrm{min}$. The ionisation mode used was electrospray negative. MRM was used for detection with the transitions $213 \rightarrow 198.2$ for PER and $165 \rightarrow 121$ for Lucinol as internal standard.

The specificity of the analytical method was controlled with blank $(\mathrm{NaCl}, 9 \mathrm{~g} / \mathrm{L})$ solution (Merck, Darmstadt Germany). The limit of quantitation (LoQ) was $2.43 \mathrm{ng} / \mathrm{mL}$. Linearity was determined between the LoQ and $1000 \mathrm{ng} / \mathrm{mL}$, with accuracy below $\pm 15 \%$, except 
at the LoQ, which was below $\pm 20 \%$. Accuracy and precision were determined at least at two quality control (QC) theoretical concentrations: low (around $20 \mathrm{ng} / \mathrm{mL}$ ) and middle (around $300 \mathrm{ng} / \mathrm{mL}$ ). All QCs remained within the acceptance criteria (accuracy $< \pm 15 \%$ ). Matrix effects and stability in buffer solutions and buffer supplemented with pig skin were evaluated at two concentrations ( 426 and $21.5 \mathrm{ng} / \mathrm{mL}$ ) in triplicate by spiking buffer solutions containing known amounts of chemical. The stability in buffer solutions spiked with PER was $98.3 \pm 7.0 \%$. A matrix effect was observed; therefore, all calibrations for this chemical were carried out in the matrix.

The penetration parameters (permeability coefficient, $K_{p}$, and flux) were determined from the curves representing the cumulative amount per unit area of skin $\left(Q t, \mu \mathrm{g} / \mathrm{cm}^{2}\right)$ as a function of time (h). The calculation was carried out using GraphPad PrismT v.7 (GraphPad Software Inc., San Diego, CA, USA).

\section{Results}

\subsection{Solubility of PER in Different Solvents}

Approximate solubility at saturation was measured for 13 solvents with the method described in the Methods section. In addition, the solubility was measured independently with LC/MS/MS methods for nine solvents to confirm the validity of the semi-quantitative approach. For these solvents, the differences in solubility values between the two different approaches were within a factor of 2, except for the highest solubility (i.e., PER in S2 and S9). This means good agreement, as shown by Table 2. Zhang and co-workers [23] have recently reported PER solubility data in propylene glycol (PG), glycerol and dimethylisosorbide (DMI). The reported values for PG are in good agreement with the results found in this study, but the data for DMI and glycerol are significantly different, which may be explained by the differences in their method, in the amount of solid excess or in the crystal form.

The solvents are grouped into three main types according to their molecular weight (MW) and polarity (see Table 2). The first group included six low-MW polar solvents; the second group included three low-MW polar "glycol" solvents; and the third group included four higher-MW non-polar solvents. Four solvents were simple two- or threecomponent solvent mixtures. The approximate solubility classification of PER at $32{ }^{\circ} \mathrm{C}$ was in agreement with its lipophilicity. PER, with a $\log P$ of 2.98 , is poorly soluble $(\sim 1 \mathrm{mg} / \mathrm{mL})$ in water and in highly non-polar organic solvents (S11-13), while it is readily soluble in semi-polar organic solvents (S2, S7, S9).

The solubility at $32{ }^{\circ} \mathrm{C}$ in Prisma buffer pH 7.4 was also measured. PER is present at this $\mathrm{pH}$ in non-ionized form; thus, the value obtained is the intrinsic solubility $\left(S_{0}\right)$. The intrinsic solubility of PER was found to be $3.45 \pm 0.01 \mathrm{mg} / \mathrm{mL}$. Ten percent of this value, $0.345 \mathrm{mg} / \mathrm{mL}$, has been selected as the target upper limit of the concentrations in the acceptor compartment to maintain a steady-state sink condition throughout the assay.

\subsection{Effect of Solvents on PAMPA Membrane Integrity}

No solvent effect was recorded on the visual appearance of the membranes after their removal and before the addition of piroxicam solution. The $\log P_{m}$ of piroxicam from aqueous solution measured across each solvent-treated membrane ranged between -3.82 and -4.81 , with a mean of $-4.25 \pm 0.30$ (Figure 1). This fits well with the reference $\log P_{m}$ value of $-4.98 \pm 0.01$ that was measured previously in an aqueous solution using this PAMPA model [22]. All permeability values of piroxicam were within one order of magnitude of the previous control $\log P_{m}$ value. Variation, i.e., the SD of the permeability values of piroxicam, provides a good indication of membrane integrity. Extreme high standard deviation would indicate membrane damage. As shown in Figure 1, the error bars are small (average $\mathrm{SD} \pm 0.08$ ), with ethanol (S2) presenting the highest variation (SD: \pm 0.23 ) and thus the largest effect on the membrane, but this SD is still acceptable, indicating an interaction of ethanol with the membrane rather than its corruption. 


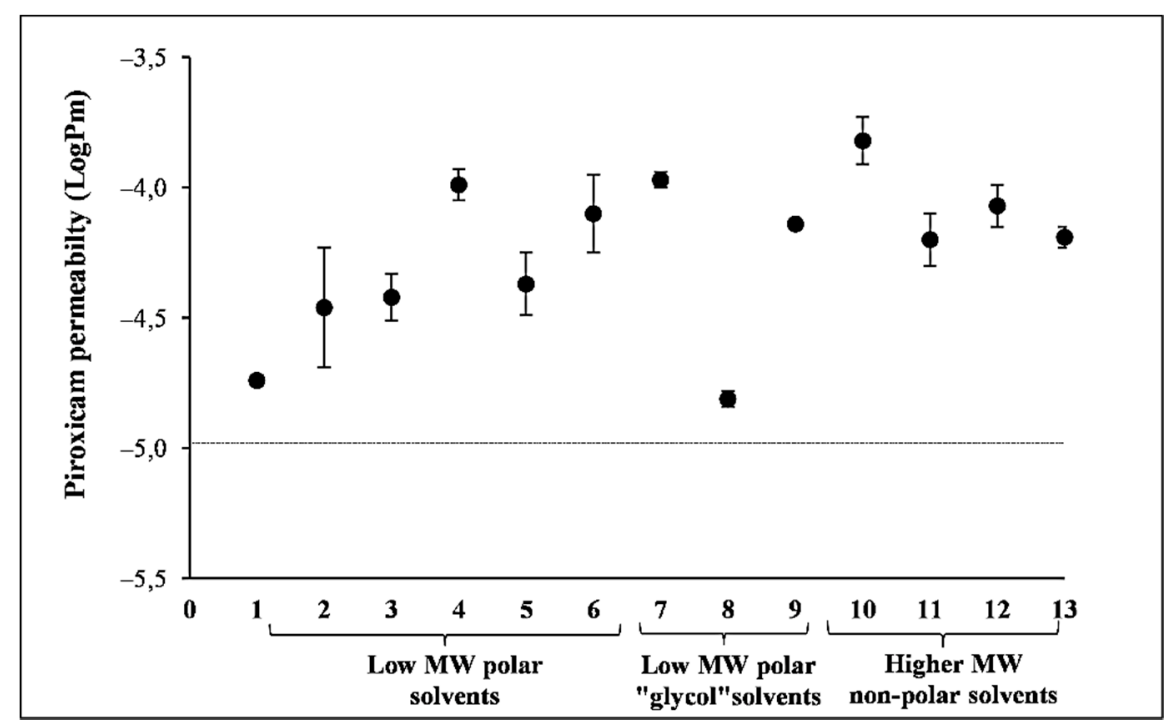

Figure 1. Effect of 13 solvents on the integrity of the Skin-PAMPA membrane using piroxicam as the model permeant. Permeability of piroxicam dissolved in water was measured after $7 \mathrm{~h}$ pre-treating membranes with each solvent. The permeability values are mean $\pm \mathrm{SD}, n=9$.

Therefore, all solvents were considered appropriate for the study, with minor signs of membrane interaction.

\subsection{Effect of Solvent on the Permeability of PER Using PAMPA}

PAMPA measurements aim to provide relevant information regarding the effect of solvents on skin permeability in a high-throughput screening format; therefore, the most characteristic parameters of the permeation process (see Table 3) were calculated from the permeated amount vs. time plot. In the case of infinite dosing conditions, the permeated amount vs. time plot is expected to be linear up until the point where the acceptor concentration is reaching the limitation of solubility or inifinite dose is no longer respected (i.e., concentration in donor compartment significantly decreased) on the example profile of PER dissolved in water on Figure 2a (solvent S1). Similar saturation curves and linear regression plots were obtained for all the solvents (see Supplementary Materials). To avoid the impact of the limitations, the first three timepoints $(7.5 \mathrm{~min}, 15 \mathrm{~min}, 30 \mathrm{~min}$ ) were selected to calculate the flux through the membrane and the lag time. Figure $2 \mathrm{~b}$ shows the result of the linear regression analyses. The linear range of the permeability profile was used for the calculation of flux (slope of the linear equation) and lag time ( $x$ value at $y=0)$. The calculated lag times (0-6 min) indicated fast membrane saturation of PER regardless of the solvent.

The relevant permeability parameters across Skin-PAMPA membranes of the nonpolar test chemical, PER, dissolved in different solvents are shown in Table 3. The different solvents had a significant impact on its permeability, whereby the $\log P_{m}$ values varied by about 1.5 orders of magnitude (which is more than the variation caused by the solvent alone). The highest permeability $\left(\log P_{m}>-1.2\right)$ was achieved when PER was dissolved in water (S1) and predominantly water-containing mixtures (S5, S6 and S8). Solvents resulting in medium permeability $\left(\log P_{m}\right.$ range -1.5 and -2.3$)$ mainly covered two chemical types: (a) higher-MW non-polar solvents such as long-chain fatty acid esters (S10) and long-chain alcohol (S11), and (b) the small polar alcohols (S2, S3) and a solvent mixture containing glycol and ethanol (S9). Solvents resulting in low permeability $\left(\log P_{m}<-2.4\right)$ of PER were low-MW polar organic solvents including dimethylisosorbide (S4) and propylene glycol (S7), in which PER was readily soluble (75 and $500 \mathrm{mg} / \mathrm{mL}$, respectively). There were two higher-MW non-polar solvents in which PER was poorly soluble $(\sim 1 \mathrm{mg} / \mathrm{mL})$, namely, apricot kernel oil (S12) and corn oil (S13). 
Table 3. Characteristic parameters of permeability of PER across Skin-PAMPA membrane for comparison when applied in different solvents.

\begin{tabular}{|c|c|c|c|c|c|c|}
\hline Code & $\begin{array}{c}C_{D} \\
{[\mathrm{mg} / \mathrm{mL}]}\end{array}$ & $\frac{J}{\left[\mu \mathrm{g} / \mathrm{cm}^{2} \times \mathrm{h}\right]}$ & $\begin{array}{l}\text { Lag Time } \\
\text { [min] }\end{array}$ & $\begin{array}{c}\text { Permeated } \\
\text { Amount }(6 \mathrm{~h}) \\
{\left[\mu \mathrm{g} / \mathrm{cm}^{2}\right]}\end{array}$ & $\begin{array}{c}A U C \\
\text { Normalized to } C_{D}\end{array}$ & $\log P_{m}$ \\
\hline S1 & 1 & $72.4 \pm 7.8$ & 1.4 & $169 \pm 4$ & 647 & $-1.12 \pm 0.06$ \\
\hline $\mathrm{S} 2$ & 500 & $12,033 \pm 252$ & 0.0 & $13,575 \pm 1710$ & 105 & $-1.62 \pm 0.01$ \\
\hline S3 & 5 & $137 \pm 22$ & 5.5 & $869 \pm 112$ & 491 & $-1.57 \pm 0.07$ \\
\hline S4 & 70 & $209 \pm 53$ & 3.6 & $1342 \pm 298$ & 55.2 & $-2.54 \pm 0.13$ \\
\hline S5 & 10 & $589 \pm 25$ & 0.9 & $1662 \pm 169$ & 569 & $-1.23 \pm 0.03$ \\
\hline S6 & 1 & $76.4 \pm 15.2$ & 0.0 & $175 \pm 22$ & 551 & $-1.13 \pm 0.07$ \\
\hline S7 & 500 & $2118 \pm 502$ & 3.8 & $11,142 \pm 729$ & 69.1 & $-2.38 \pm 0.11$ \\
\hline S8 & 10 & $570 \pm 5$ & 1.1 & $1772 \pm 98$ & 595 & $-1.24 \pm 0.01$ \\
\hline S9 & 500 & $10,846 \pm 326$ & 1.4 & $14,926 \pm 2431$ & 114 & $-1.66 \pm 0.01$ \\
\hline S10 & 70 & $377 \pm 36$ & 3.0 & $749 \pm 92$ & 38.2 & $-2.27 \pm 0.04$ \\
\hline S11 & 1 & $7.18 \pm 1.6$ & 3.9 & $19 \pm 0.4$ & 64.5 & $-2.16 \pm 0.08$ \\
\hline S12 & 1 & $2.45 \pm 0.57$ & 3.3 & $13 \pm 1.9$ & 36.7 & $-2.63 \pm 0.07$ \\
\hline S13 & 1 & $3.26 \pm 0.41$ & 1.4 & $13 \pm 1.3$ & 38.6 & $-2.48 \pm 0.06$ \\
\hline
\end{tabular}

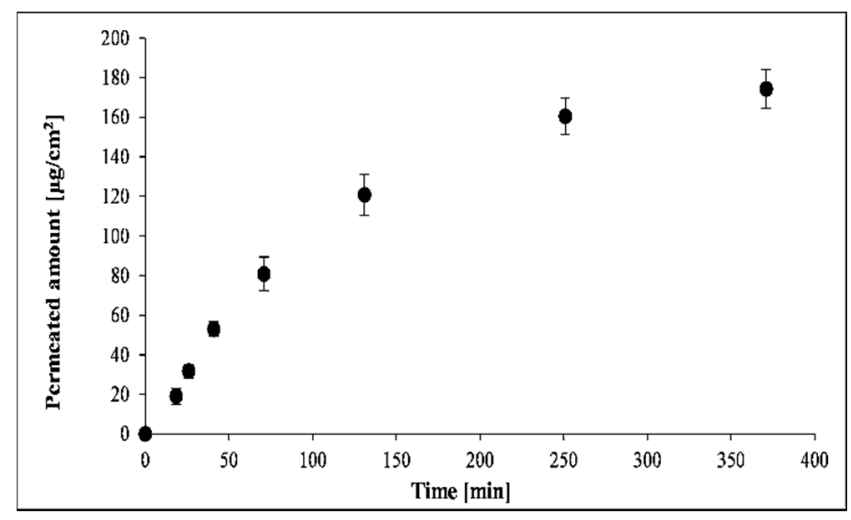

(a)

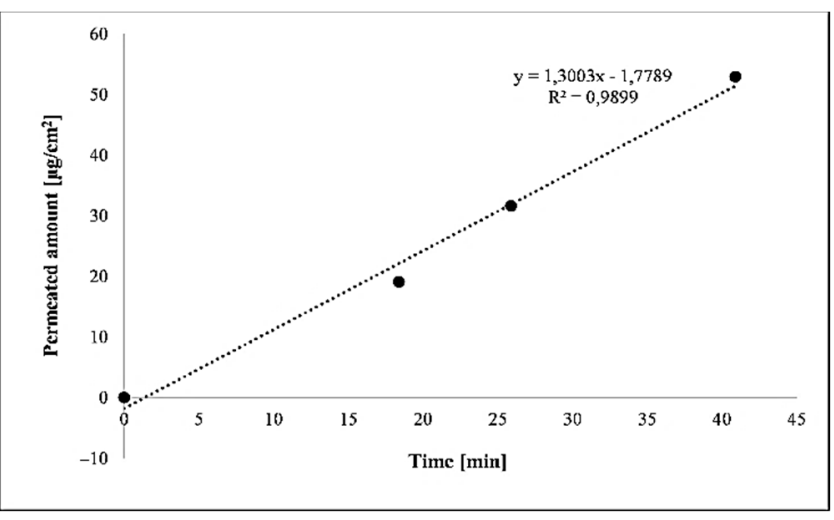

(b)

Figure 2. (a) The permeability profile of PER dissolved in water using Skin-PAMPA; (b) The linear regression curve used for the calculation of flux.

\subsection{Comparison of the Permeability of PER Using Skin-PAMPA vs. Pig Skin}

The permeability of PER across pig skin was determined for nine solvents (see data in Table 4) and compared with the values measured in the PAMPA. These included mostly low-MW polar solvents (S1, S2, S4, S5, S6), three low-MW polar "glycols" (S7, S8, S9) and one high-MW non-polar solvent (S10).

Table 4. Characteristic parameters of permeability of PER across pig ear skin in different solvents.

\begin{tabular}{ccccc}
\hline Code & $\mathbf{C}_{\mathbf{D}}[\mathbf{m g} / \mathbf{m L}]$ & $\mathbf{J}\left[\boldsymbol{\mu g} / \mathbf{c m}^{\mathbf{2}} \times \mathbf{h}\right]$ & $\begin{array}{c}\text { Permeated Amount } \\
\mathbf{( 1 6} \mathbf{h})\left[\boldsymbol{\mu g} / \mathbf{c m}^{\mathbf{2}}\right]\end{array}$ & $\log K_{\boldsymbol{p}}$ \\
\hline S1 & 1 & $25 \pm 9.6$ & $261 \pm 100$ & $-1.71 \pm 0.32$ \\
S2 & 368 & $20 \pm 15$ & $98 \pm 67$ & $-4.26 \pm 0.51$ \\
S4 & 60 & $0.08 \pm 0.024$ & $1.09 \pm 0,39$ & $-5.88 \pm 0.19$ \\
S5 & 8 & $61 \pm 18$ & $515 \pm 203$ & $-1.97 \pm 0.34$ \\
S6 & 1 & $7.5 \pm 3$ & $100 \pm 37$ & $-2.16 \pm 0.26$ \\
S7 & 350 & $20 \pm 17.6$ & $147 \pm 120$ & $-4.24 \pm 0.81$ \\
S8 & 51 & $98 \pm 50$ & $516 \pm 109$ & $-1.72 \pm 0.34$ \\
S9 & 373 & $40 \pm 15$ & $320 \pm 149$ & $-3.97 \pm 0.24$ \\
S10 & 75 & $8 \pm 3.5$ & $54 \pm 34$ & $-3.97 \pm 0.29$ \\
\hline
\end{tabular}


Figure 3a shows the comparison of flux between Skin-PAMPA and pig skin (in increasing values for pig skin). The absolute values of flux in Skin-PAMPAs were higher than for pig skin, but a comparison can be done by showing the values on different y-axes. In four solvents (S1, S5, S6 and S8), the difference was within one order of magnitude despite potential underestimation of permeability coefficient using Skin-PAMPA. In contrast, for three solvents (S4, S7 and S10), Skin-PAMPA overestimated PER flux by more than two orders of magnitude. There were two clear outliers in the correlation: solvents S2 and S9.

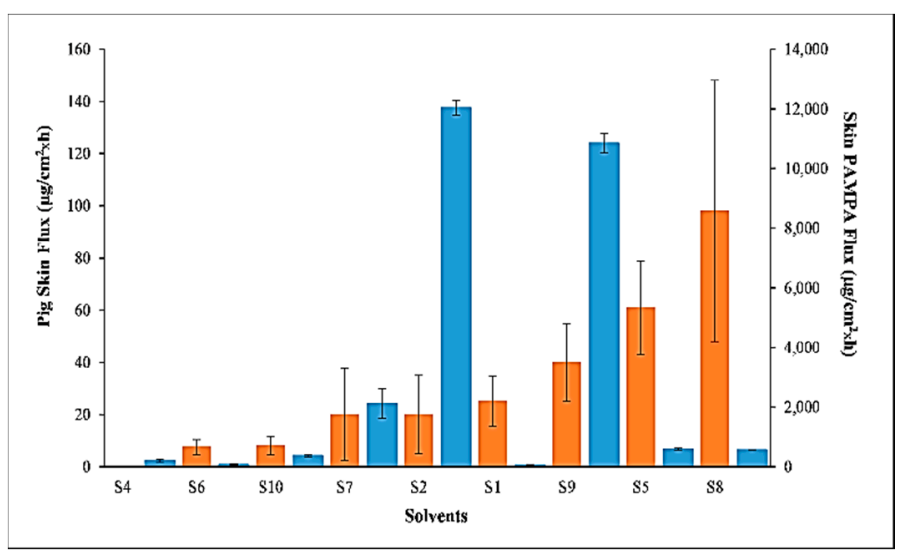

(a)

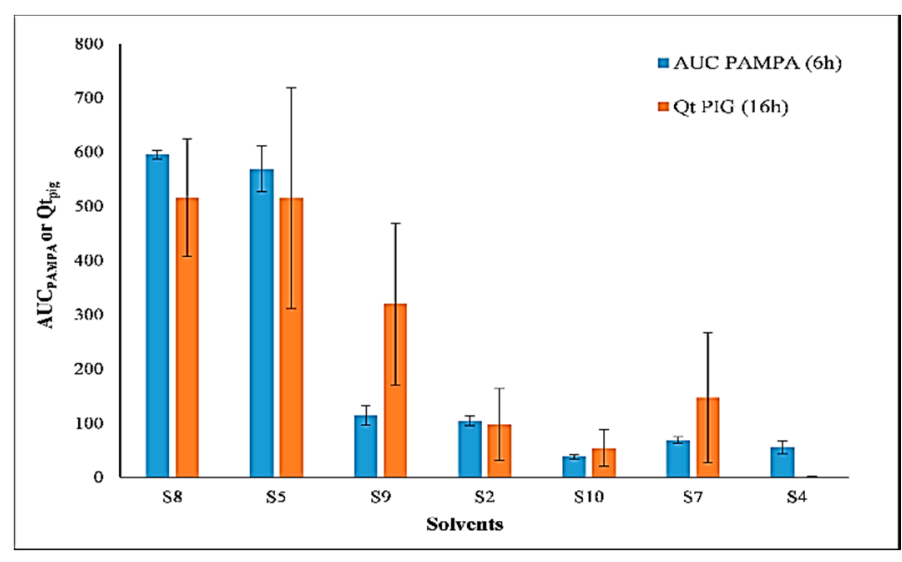

(b)

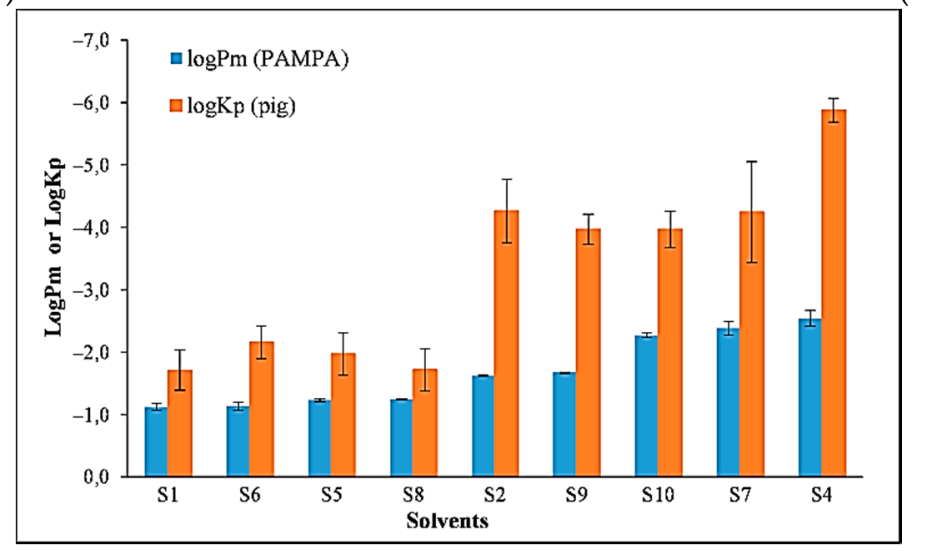

(c)

Figure 3. (a) Comparison of the permeability of PER in different solvents across Skin-PAMPA membranes (blue bars) and pig skin (orange bars), expressed as flux (no close correlation); (b) permeated amount $\left(R^{2}=0.834, n=7\right)$; (c) permeability coefficient, $\log P_{m}$ and $\log K_{p}$ for Skin PAMPA and pig skin, respectively $\left(R^{2}=0.844, n=9\right)$. All values are mean $\pm \mathrm{SD}$.

Permeated amount at $6 \mathrm{~h}$, expressed as $A U C_{P A M P A}$ (calculated by integration of the permeability profile between 0 and $6 \mathrm{~h}$ and normalized to the donor concentration) for SkinPAMPA and $Q t_{\text {pig skin }}$ at $16 \mathrm{~h}$ for pig skin, was also correlated. The comparison between the amounts of PER detected in the acceptor compartments in PAMPA and pig skin provides a much closer trend than flux data comparison (Figure 3b). Only two outliers were detected, namely S1 and S6 (their data not shown), and for seven solvents, the correlation coefficient is $R^{2}=0.843$, which can be considered reasonable.

The best correlation between the two models occurred when the data were expressed as the $\log P_{m}$ for PAMPA and $\log K_{p}$ for pig skin assays (Figure 3c). Since these values were normalised to the concentration in the donor compartment, they can be plotted on the same $y$-axis. The calculation of the two permeability values applied the same mathematical equation derived from the relationship $P_{m}=J / C_{D}$, where $J$ is the flux, and $C_{D}$ is the initial donor concentration. As expected, when comparing a single membrane with multiple layered 700-1200 $\mu \mathrm{m}$ thick native pig skin, the permeability coefficients in Skin-PAMPAs were higher than pig skin. The biggest differences between values from the Skin-PAMPA 
and pig skin models were when PER was dissolved in dimethylisosorbide (S4) and ethanol (S2). Nevertheless, there was a good correlation between the two values for the nine solvents, with an $R^{2}$ of 0.844 . In fact, there was no outlier in this correlation between the two models.

\section{Discussion}

The applicability of the Skin-PAMPA was investigated as a screening tool to differentiate between the permeabilities of a model compound dissolved in different solvents. The study was focused on the behavior of the Skin-PAMPA membrane when an active ingredient was applied in different solvents that are applied routinely in the cosmetic industry. PER was selected as the model compound of non-polar chemicals, which is a well-studied compound with a range of physico-chemical properties already available in the literature.

The Skin-PAMPA measures the permeability of solutions that are close to their saturated concentrations. Therefore, we measured the solubility of PER in each solvent. Since an exact solubility was difficult to measure for some solvents, solubility was classified in five main categories and four sub-categories based on visual assessments. The classification of solubility based on visual evaluation (of any undissolved particles of the solid) correlated very well with that measured using LC/MS/MS methods for the pig skin assays (Table 2). PER was soluble in the solvents tested, and the data were in agreement with its moderate lipophilicity, such that it was poorly soluble in highly non-polar and highly polar solvents, while it was best dissolved in semi-polar solvents and their mixtures.

When conducting skin penetration assays, technical aspects that could impact results should be considered. One of the important aspects to consider is that the permeated amount should not exceed $10 \%$ of the applied dose to provide accurate permeability values. When it exceeds $10 \%$ of the applied dose, the permeability coefficient may be underestimated. A second aspect relates to the effect of the solvent itself upon the integrity of the membrane. In order to investigate the direct effect of solvents on the Skin-PAMPA membrane integrity, the membrane was pre-treated with each solvent (in the absence of chemical), and after removing them, piroxicam was measured as the test permeant [22]. The permeability of piroxicam was increased depending on pre-treatment with the solvents, possibly due to the partitioning of piroxicam $(\log P=1.71)$ into the residual solvent layer at the surface of the membrane, which provided a higher surface concentration. However, the variation in permeability of piroxicam in the different solvents was found to be of minor amplitude, which indicates that the membrane integrity was intact, so the solvents were not damaging the membrane structure, or at least all changes were stable by the end of the incubation.

The Skin-PAMPAs were performed with a $6 \mathrm{~h}$ incubation in each studied solvent solution, which allowed high-throughput evaluation. The permeability of PER was significantly affected by the solvent in which it was dissolved, such that the $\log P_{m}$ spanned about 1.5 orders of magnitude. The permeability could be divided into three classes: low $\left(\log P_{m}<-2.4\right)$, medium $\left(\log P_{m}\right.$ from -2.3 to -1.5$)$ and $h i g h\left(\log P_{m}>1.2\right)$. Solutions of PER provided examples for all classes.

Great attention had to be devoted to the following factors, which are the limitations of this method. Appropriate precise pipetting is essential in this technique. Compounds with excessively high or low permeation properties cannot be measured. Applying viscous solvents can be challenging because the application of solvents to PAMPA plate is a timeconsuming process, so correction for the time factor needs to be implemented during the evaluation of the results. Finally, the tension of the solvents can also be a limiting factor, since the concentration of high-tension solutions can be modified during the experiment, leading to invalid permeability results.

To determine whether the Skin-PAMPA model provides an accurate estimation of permeability, the results were compared with those obtained from penetration studies using pig skin. Permeation potential can be expressed in a number of ways: the amount 
penetrated in a finite time $(Q t)$; flux $(J)$, representing the mean mass transfer through the membrane; and the permeability coefficient $\left(P_{m}\right.$ or $\left.K_{p}\right)$, reflecting the rate of penetration through the membrane. Therefore, the comparisons between Skin-PAMPA and pig skin permeability were also used to identify the best parameter to differentiate the permeability of the chemical in different solvents. When the ranking of the permeation potential of PER in different solvents was expressed as the flux, there was a poor correlation between values from the Skin-PAMPA and pig skin assays. Better correlation was found between the amounts penetrated ( $A U C_{P A M P A}$ vs. $\left.Q t_{\text {pig skin }}\right)$, but two solvents were outliers. The best correlation was achieved when permeability was expressed as log of the permeability coefficient, $\log K_{p}$ or $\log P_{m}$. Both assays indicated that the permeability of PER in solvents S2, S4, S7, S9 and S10 was higher than when it was dissolved in the other four solvents. The biggest differences between values from the Skin-PAMPA and pig skin models were observed when PER was dissolved in dimethylisosorbide (S4) and in ethanol (S2). These differences were not due to the solvent per se, since this was excluded in the pre-tests; however, the combination of PER and solvent may have disrupted the PAMPA membrane structure, resulting in a higher permeability. The difference in the permeability coefficients between the two models was much less when these solvents were in mixtures with water containing a lower concentration of the organic component (e.g., S6 and S5).

Notably, the ranking of the permeation potential was different based on the expression of the data. For example, S1 and S6 received low rankings when data were expressed as the amount penetrated or the flux, but they were ranked among the highest ones when the $\log K_{p}$ or $\log P_{m}$ were used. Since flux is the product of permeability and the donor concentration, and the concentrations tested were near to saturated values, a higher solubility in the donor compartment may be expected to result in a proportional increase in the flux. This was generally reflected in the Skin-PAMPA flux values for PER (Figure 4a,b), albeit with some exceptions (e.g., the solubility of PER in water and corn oil were both about $1 \mathrm{mg} / \mathrm{mL}$, but the flux was 17 -fold lower in corn oil than when dissolved in water) that indicate the importance of complex solubility/dissolution and permeation studies. By contrast, the permeability coefficient, $\log P_{m}$ or $\log K_{p}$, is independent of the concentration used, making it a more appropriate measure of permeability for chemicals that have large differences in solubility in different solvents.

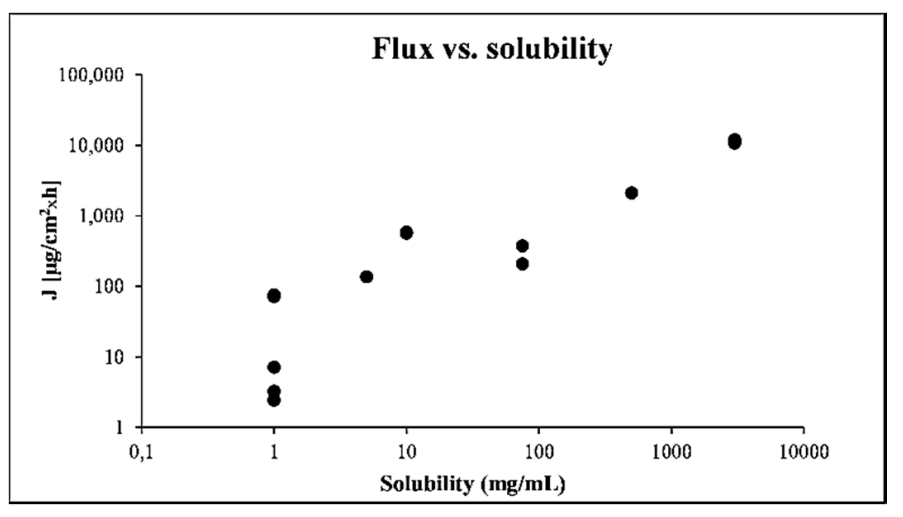

(a)

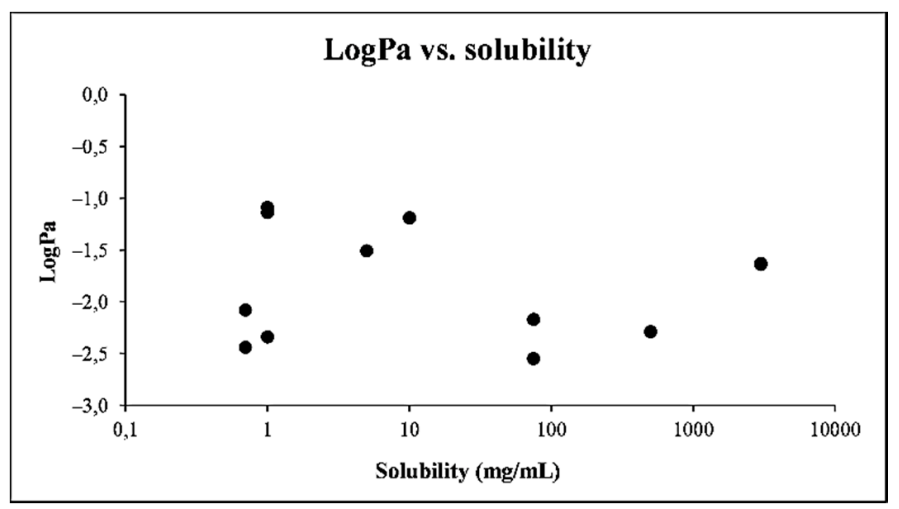

(b)

Figure 4. (a) Comparison of the solubility of PER with flux; (b) $\log P_{m}$. All values are mean.

\section{Conclusions}

In conclusion, the Skin-PAMPA allows the evaluation of the permeability of model compound dissolved in multiple and widely varying solvent types, from highly polar to highly non-polar, as well as mixtures of solvents. It was possible to classify the permeability of PER into 3 categories: low, medium and high. The most appropriate parameter for the comparison of permeability was the permeability coefficient, $\log P_{m}$ or $\log K_{p}$, which is independent of the concentration of the solution applied. This is particularly important 
for chemicals that have large differences in solubility. The comparison of the relative permeability of PER in different solvents was confirmed by comparing the permeability coefficients with those measured in pig skin permeability assays. Our results support the use of the Skin-PAMPA for screening the suitability of different solvents for non-polar test compounds at early stages of product development.

Supplementary Materials: The following are available online at https:/ /www.mdpi.com/article/10 .3390 / pharmaceutics13111758/s1, Figure S1: The permeability profile of PER dissolved in ethanol using Skin-PAMPA, Figure S2: The permeability profile of PER dissolved in glycerol using SkinPAMPA, Figure S3: The permeability profile of PER dissolved in dimethylisosorbide using SkinPAMPA, Figure S4: The permeability profile of PER dissolved in water/ethanol-80:20 (w/w) using Skin-PAMPA, Figure S5: The permeability profile of PER dissolved in water/dimethylisosorbide90:10 $(w / w)$ using Skin-PAMPA, Figure S6: The permeability profile of PER dissolved in propylene glycol using Skin-PAMPA, Figure S7: The permeability profile of PER dissolved in water:propylene glycol-80:20 $(w / w)$ using Skin-PAMPA, Figure S8: The permeability profile of PER dissolved in wa-ter/propylenglycol/ethanol-10:30:60 $(w / w / w)$ using Skin-PAMPA, Figure S9: The permeability profile of PER dissolved in capric/caprylic triglyc-erides using Skin-PAMPA, Figure S10: The permeability profile of PER dissolved in octyl dodecanol using Skin-PAMPA, Figure S11: The permeability profile of PER dissolved in apricot kernel oil using Skin-PAMPA, Figure S12: The permeability profile of PER dissolved in corn oil using Skin-PAMPA.

Author Contributions: Conceptualization, B.S., C.J. and S.G.; methodology, D.V. and S.K.; software, S.K.; formal analysis, A.M., C.J., V.B. and P.M.; investigation, D.V.; data curation, A.M., P.M. and S.K.; writing—original draft preparation, V.B. and S.K.; writing—review and editing, S.G., K.T.-N. and B.S.; visualization, V.B.; supervision, K.T.-N.; project administration, V.B.; funding acquisition, S.G. and B.S. All authors have read and agreed to the published version of the manuscript.

Funding: This research received no external funding.

Institutional Review Board Statement: Not applicable.

Informed Consent Statement: Not applicable.

Data Availability Statement: All data can be provided by the authors upon request. No publicly accessable archive storage is available.

Acknowledgments: We wish to thank N. Hewitt for helping in writing the manuscript and B. Bernard for his critical reading of the manuscript.

Conflicts of Interest: A.M., C.J. and S.G. are employees of L'Oréal. L'Oréal had no role in the design of the study; in the collection, analyses, or interpretation of data; in the writing of the manuscript; or in the decision to publish the results.

\section{References}

1. Howes, D.; Guy, R.H.; Hadgraft, J.; Heylings, J.; Hoeck, U.; Kemper, F.; Maibach, H.; Marty, J.; Merk, H.; Parra, J.; et al. Methods for assessing percutaneous absorption-The report and recommendations of ECVAM workshop 13. ATLA-Altern. Lab. Anim. 1996, 24, 81-106. [CrossRef]

2. Prausnitz, M.R.; Langer, R. Transdermal drug delivery(Review). Nat. Biotechnol. 2008, 26, 1261-1268. [CrossRef]

3. Wiedersberg, S.; Guy, R.H. Transdermal drug delivery: 30 + years of war and still fighting! J. Control. Release 2014, 190, 150-156. [CrossRef]

4. Scientific Committee on Consumer Safety Basic criteria for the in vitro assessment of dermal absorption of cosmetic ingredients. Eur. Comm. 2010, SCCS/1358, 1-14.

5. OECD Test No. 428: Skin Absorption: In Vitro Method. In OECD Guidelines for the Testing of Chemicals, Section 4; OECD: Paris, France, 2004.

6. Kraeling, M.E.K.; Bronaugh, R. In vitro percutaneous absorption of alpha hydroxy acids in human skin. J. Soc. Cosmet. Chem. 1997, 48, 187-197.

7. Gerstel, D.; Jacques-Jamin, C.; Schepky, A.; Cubberley, R.; Eilstein, J.; Grégoire, S.; Hewitt, N.; Klaric, M.; Rothe, H.; Duplan, H. Comparison of protocols for measuring cosmetic ingredient distribution in human and pig skin. Toxicol. Vitr. 2016, 34, 153-160. [CrossRef] [PubMed] 
8. Wargniez, W.; Jungman, E.; Wilkinson, S.; Seyler, N.; Grégoire, S. Inter-laboratory skin distribution study of 4-n-butyl resorcinol: The importance of liquid chromatography/mass spectrometry (HPLC-MS/MS) bioanalytical validation. J. Chromatogr. B 2017, 1060, 416-423. [CrossRef]

9. Lotte, C.; Patouillet, C.; Zanini, M.; Messager, A.; Roguet, R. Permeation and Skin Absorption: Reproducibility of Various Industrial Reconstructed Human Skin Models. Ski. Pharmacol. Appl. Ski. Physiol. 2002, 15, 18-30. [CrossRef] [PubMed]

10. Schäfer-Korting, M.; Bock, U.; Diembeck, W.; Düsing, H.-J.; Gamer, A.; Haltner-Ukomadu, E.; Hoffmann, C.; Kaca, M.; Kamp, H.; Kersen, S.; et al. The Use of Reconstructed Human Epidermis for Skin Absorption Testing: Results of the Validation Study. Altern. Lab. Anim. 2008, 36, 161-187. [CrossRef] [PubMed]

11. De Jager, M.W.; Gooris, G.; Ponec, M.; Bouwstra, J. Lipid mixtures prepared with well-defined synthetic ceramides closely mimic the unique stratum corneum lipid phase behavior. J. Lipid. Res. 2005, 46, 2649-2656. [CrossRef]

12. Ottaviani, G.; Martel, A.S.; Carrupt, P.-A. Parallel Artificial Membrane Permeability Assay: A New Membrane for the Fast Prediction of Passive Human Skin Permeability. J. Med. Chem. 2006, 49, 3948-3954. [CrossRef]

13. Joshi, V.; Brewster, D.; Colonero, P. In vitro diffusion studies in transdermal research: A synthetic membrane model in place of human skin. Drug Dev. Deliv. 2012, 12, 40-42.

14. Provin, C.; Nicolas, A.; Grégoire, S.; Fujii, T. A Microfluidic Diffusion Cell for Fast and Easy Percutaneous Absorption Assays. Pharm. Res. 2015, 32, 2704-2712. [CrossRef]

15. Sinkó, B.; Garrigues, T.M.; Balogh, G.T.; Nagy, Z.K.; Tsinman, O.; Avdeef, A.; Takács-Novák, K. Skin-PAMPA: A new method for fast prediction of skin penetration. Eur. J. Pharm. Sci. 2012, 45, 698-707. [CrossRef] [PubMed]

16. Baroni, A.; Buommino, E.; De Gregorio, V.; Ruocco, E.; Ruocco, V.; Wolf, R. Structure and function of the epidermis related to barrier properties. Clin. Dermatol. 2012, 30, 257-262. [CrossRef]

17. Karadzovska, D.; Riviere, J.E. Assessing vehicle effects on skin absorption using artificial membrane assays. Eur. J. Pharm. Sci. 2013, 50, 569-576. [CrossRef] [PubMed]

18. Haq, A.; Goodyear, B.; Ameen, D.; Joshi, V.; Michniak-Kohn, B. Strat-M®synthetic membrane: Permeability comparison to human cadaver skin. Int. J. Pharm. 2018, 547, 432-437. [CrossRef] [PubMed]

19. Sinkó, B.; Vizserálek, G.; Novák, K.T. Skin PAMPA: Application in practice. ADMET DMPK 2015, 2, 191-198. [CrossRef]

20. Balázs, B.; Vizserálek, G.; Berkó, S.; Budai-Szúcs, M.; Kelemen, A.; Sinkó, B.; Takács-Novák, K.; Szabó-Révész, P.; Csányi, E. Investigation of the Efficacy of Transdermal Pene-tration Enhancers Through the Use of Human Skin and a Skin Mimic Artificial Membrane. J. Pharm. Sci. 2016, 105, 1134-1140. [CrossRef]

21. Vizserálek, G.; Balogh, T.; Takács-Novák, K.; Sinkó, B. PAMPA study of the temperature effect on permeability. Eur. J. Pharm. Sci. 2014, 53, 45-49. [CrossRef]

22. Vizserálek, G.; Berkó, S.; Tóth, G.; Balogh, R.; Budai-Szúcs, M.; Csányi, E.; Sinkó, B.; Takács-Novák, K. Permeability test for transdermal and local therapeutic patches using Skin PAMPA method. Eur. J. Pharm. Sci. 2015, 76, 165-172. [CrossRef] [PubMed]

23. Zhang, Y.; Sil, B.C.; Kung, C.; Hadgraft, J.; Heinrich, M.; Sinko, B.; Lane, M.E. Characterization and topical delivery of phenylethyl resorcinol. Int. J. Cosmet. Sci. 2019, 41, 479-488. [CrossRef]

24. Hadgraft, J.; Guy, R.H. Feasibility Assessment in Topical and Transdermal Delivery: Mathematical Models and in Vitro Studies. In Transdermal Drug Delivery; CRC Press: Boca Raton, FL, USA, 2002; pp. 1-23.

25. OECD Test No. 105: Water Solubility. In OECD Guidelines for the Testing of Chemicals, Section 1; Organisation for Economic Co-Operation and Development (OECD): Paris, France, 1995.

26. Baka, E.; Comer, J.E.; Takács-Novák, K. Study of equilibrium solubility measurement by saturation shake-flask method using hydrochlorothiazide as model compound. J. Pharm. Biomed. Anal. 2008, 46, 335-341. [CrossRef]

27. Avdeef, A.; Fuguet, E.; Llinas, A.; Ràfols, C.; Bosch, E.; Völgyi, G.; Verbic, T.; Boldyreva, E.; Takács-Novák, K. Equilibrium solubility measurement of ionizable drugs—consensus recommendations for improving data quality. ADMET DMPK 2016, 4, 117-178. [CrossRef]

28. Takács-Novák, K.; Józan, M.; Hermecz, I.; Szász, G. Lipophilicity of antibacterial fluoroquinolones. Int. J. Pharm. 1992, 79, 89-96. [CrossRef]

29. Takács-Novák, K.; Józan, M.; Szász, G. Lipophilicity of amphoteric molecules expressed by the true partition coefficient. Int. J. Pharm. 1995, 113, 47-55. [CrossRef] 